\title{
GRB observations with INTEGRAL and XMM
}

\section{B. McBreen}

Department of Experimental Physicst, University College Dublin, Dublin, Ireland email: Brian.McBreen@ucd.ie

\begin{abstract}
The results obtained during the first three years of operation of INTEGRAL will be reviewed. Over 30 GRBs have been localised by the INTEGRAL burst alert system (IBAS) and the coordinates distributed. The follow-up observations with XMM-Newton and large telescopes have led to many interesting results. A comparison will also be made between the sensitivities of INTEGRAL and SWIFT.
\end{abstract}

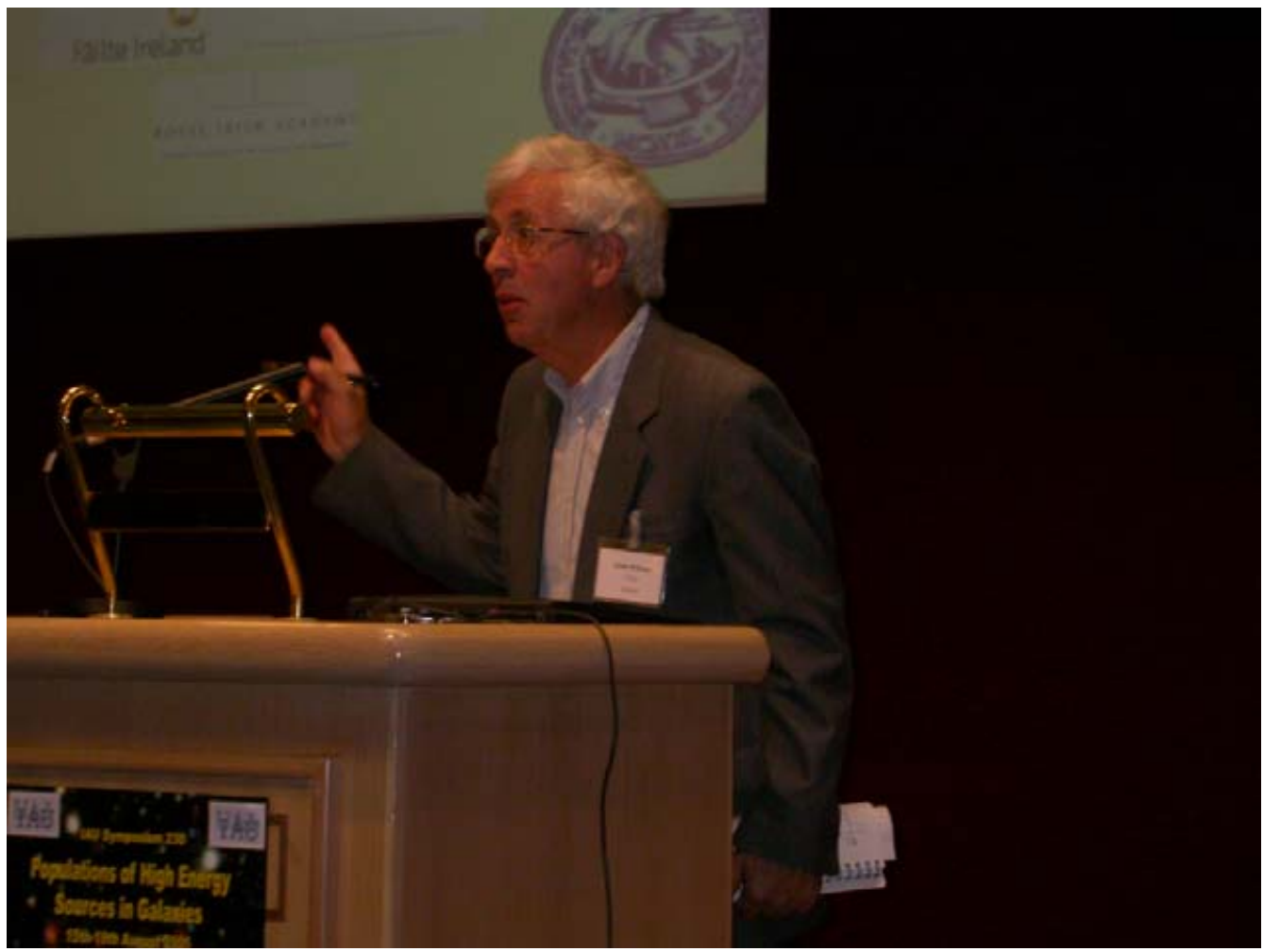

Brian McBreen pointing out room safety regulations.

$\dagger$ Now: UCD School of Physics, Science Centre North, UCD. 